\title{
Improving the Performance of the Prediction by Machine Learning Algorithms
}

\author{
M Srinivas Reddy, Kanaka Durga B, A Damodar
}

\begin{abstract}
: in the course of latest a long term, system gaining knowledge of $(M L)$ has superior from the task of barely any laptop devotees abusing the plausibility of desktops figuring out a way to play around, and a chunk of mathematics (facts) that superb occasionally belief to be computational methodologies, to a free research area that has now not just given the essential base to measurable computational requirements of studying systems, yet moreover has created wonderful calculations which can be generally carried out for content material material translation, layout acknowledgment, and a numerous excellent commercial enterprise features or has precipitated a simply one among a type studies eagerness for data removal to differentiate wearing a veil regularities or abnormality within group facts that developing via way of next. This thesis centers round clarifying the concept and development of device learning, a portion of the mainstream device gaining knowledge of calculations and attempt to reflect onconsideration on 3 most ordinary calculations relying on a few essential thoughts. Sentiment140 dataset turn out to be utilized and execution of each estimate concerning getting ready time, expectation time and precision of forecast had been archived and analyzed.
\end{abstract}

KEYWORDS: Accuracy, Algorithm, engine knowledge Data, Training.

\section{INTRODUCTION}

Simulated intelligence as a perspective that might suggest picking up with past comprehension (which for this circumstance is past data) to develop future execution. The only point of convergence of this fields is customized knowledge techniques. Learning insinuates adjustment or development of computation subject to past "familiarity" normally with no outside help from being. While arranging a mechanism (an item system), the product engineer reliably has a specific explanation at the highest point of the need list. For instance, consider J. K. Rowling'sHarry Potter Series or RobertGalbraith's Cormoran Strike Series. To avow the case that it was without a doubtRowling who had made those books below the nameGalbraith, two masters was secured by The London Sunday Times and utilize ForensicMLthey had the choice to show that case was substantial. They improve an AI estimation and "arranged" it with Rowling's similarly as various columnists making advisers for search for and get acquainted with the shrouded models and a short time later "test" the booksbyGalbraith.

Revised Manuscript Received on December 22, 2019.

M Srinivas Reddy, Asst. Professor, Dept. of CSE., Malla Reddy Engineering College for Women, Hyderabad, India.

Kanaka Durga B, Asst. Professor, Dept. of CSE., Malla Reddy Engineering College for Women, Hyderabad, India.

A Damodar, Asst. Professor, Dept. of CSE., Malla Reddy Engineering College for Women, Hyderabad, India.
The estimation contemplated that Rowling's and Galbraith's forming composed the mainly in a couple of perspectives.

So instead of organizing a computation to address the issue really, utilize MachineLearning, a pro search for a strategy during with machine, i.e., the figuring determination think about every own answer subject to the model or getting ready instructive assortment provided for it from the outset.

\section{MACHINE GAINING KNOWLEDGE OF: INTERSECTION OF FACTS AND LAPTOP TECHNOLOGICAL KNOW-HOW}

Computerized reasoning were the unimaginable outcomes when ComputerScience and Statistics consolidated. Programming structuring bases on construction machines that manage unequivocal issues, and tries to perceive if issues are reasonable in any capacity whatsoever. The standard methodology that Statistics essentially utilizes is information actuating, displaying estimates and assessing suffering nature of the terminations. The portraying ideaof Machine Learning is somewhat novel yet generally subject to both in any case. Disregarding the way that Computer Science revolve around physically programming PCs, MLaddressesthe issue of getting PCs to re-program themselves at whatever point displayed to new information subject to some major learning techniques gave. Then again, Statistics rotates around information inference and likelihood, Machine Learning merges extra worries over the credibility and sufficiency of structures and figurings to process those information, upsetting several learning assignments into an irrelevant one and execution measures.

\section{A. BEING LEARNING OR MACHINE LEARNING}

The $3^{\text {rd }}$ research zone emphatically identified with ML is an assessment of being and creature character in NeurosciencePsychology, or related fields. The specialists proposed that how a machine could get for a reality most clearly would not be commonly not actually equal to how a creature or a being character learns for time and experience. Regardless, the appraisal focused on managing AI issues utilizing learning methodologies for being cerebrum didn't yield a lot of promising outcome so far than the gets some information about worried over quantifiable - computational way of thinking. This may be an immediate aftereffect of the way wherein that being or creature cerebrum science remains not absolutely reasonable to date. Notwithstanding these issues, support between being learning and AI is stretching out for AI is being utilized to clarify several learning strategies finding in being or creatures. 


\section{Improving the Performance of the Prediction by Machine Learning Algorithms}

For instance, AI method for brief distinction were proposed to clarify neural flag in creature knowledge. If it is truly expected that this joint effort is to develop basically in coming years.

\section{B. DATA MINING, ARTIFICIAL} INTELLIGENCE AND MACHINE LEARNING

After a short time, these three controls are so bound and covering that it's going to draw a limit or development among the three. To put it consequently, these three fields are productively related and a blend of these approaches may be utilized as a procedure to pass on consistently able and sensitive outputs. Generally, Data mining is fundamentally about translating any sort of information, yet it sets up the structure for both man-made reasoning and AI. In every practical sense, it test data from different sources similarly as it assessments and sees model and affiliations that exists in those data that would have been hard to disentangle physically. Thusly, information mining is verifiably not an insignificant technique to show a theory yet system for drawing suitable hypotheses. That mined information and the taking a gander at models and speculations might be used the reason behind both AI and man-made discernment. Man-made mindfulness might be completely portrayed asmachinesthose having the choice to manage a given issue solely with no being mediation. The approaches are notprogrammed direct into the structure yet the critical information and the AI deciphering that information produce an answer free from some other individual. The understanding that goes underneath is only an information mining figuring. Recreated insight grasps raise the system to a pushed level by giving the information major to a machine to prepare and adjust reasonably when displayed to new information. This is known as "preparing". It centers onextracting data from in a general sense largesets of information, and therefore sees and perceives fundamental models utilizing different exact measures to improve its capacity to decipher new information and produce dynamically persuading outcomes. Plainly, two or three parameters ought to be "tuned" close to the beginning levelfor better capability. Computerized reasoning is thefoothold of man-made reasoning. It is presumably not going to structure any machinehaving abilitiesassociated with data, similar to language or vision, to arrive immediately. That errand would have been in every practical sense hard to appreciate. In addition, a framework can not be considered completely astute on the off chance that it came up short on the capacity to take in and improve from its past exposures.

\section{PRESENT RESEARCH QUESTIONS\& RELATED WORK}

The Several applications referenced before proposes broad advancementso far in ML counts and their pivotal speculation. The request is uncovering a couple of way, testing an extent of learning issues. ML is a tremendous control and over ongoing decades different researchers have remembered their works for this field. The rundown of these works are countably interminable and referencing each work is degree as this paper. Howeverthis paper portrays the rule ask about question that are being looked for after at display and give reference tosome of the continuous famous arrangements with that endeavor.

\section{A. USING UNLABELLED DATA IN SUPERVISED LEARNING}

Direct getting to know counts assessed the affiliation with functions and names through portraying an estimation $\mathrm{f}: \mathrm{X}$ $\rightarrow \mathrm{Y}$ used for a particular group of pre-checked planning facts $\{\square \mathrm{xi}, \mathrm{yi} \square\}$. the rule of thumb challenge on this approach is pre-checked statistics isn't for each situation right away available. So before making use of Supervise categorization, data need to be preprocesse, clean and named the utilize of independent studying, incorporate removal, dimensionality decline, and many others there by means of including to the overall cost.This move in cost can be diminished sufficiently if the supervise count can utilize unlabelled facts (e.g., pictures) as well. Inquisitively, in various excellent events of learning issues with additional assumptions, unlabelled data can without a doubt be advocated to improve the typical precision of oversaw learning. Like,consider orchestrating site pages or perceiving spam messages. At present powerful researchers are really considering new computations or new learning issues to mishandle unlabelled data capably.

\section{B. \\ TRANSFERRINGTHE \\ EXPERIENCE \\ LEARNING}

In various certified issue, the directed figuring may incorporate learning a gathering of related limits (e.g., assurance functionsfor crisis centers over the globe) rather than a lone limit. Notwithstanding whether the discovering functionsfor different urban regions (e.g., Kolkata and London) are endeavored to be commonly uncommon, a couple of shared qualities are anticipated moreover. ML algorithmslike different leveled Bayesian methodsgive one technique that expect the learning parameters of both the limits, state for Kolkata and London independently, havesome standard prior probabilities, and grants the data from different city therapeutic facilities to over ruler elevant priors as becoming. The subtlety further augmentations when the trade among the limits are exacerbated.

\section{C.LINKING EXCLUSIVE ML ALGORITHMS}

Different ML counts has been displayed and researched to different spaces. One way of investigate intends to determine the achievable associations during an ebb and flow Machine Learning counts, \& proper case or circumstances to utilize a exacting figuring. regard as, speculations two controlled gathering figurings, NaiveBayes and Logistic Regressions. Them two technique various educational assortments especially, anyway their indistinguishable quality can be indicated when realized to express sorts of getting ready information. At the point when all is said in done, the concept understanding of ML computations, their union feature, and their particular efficiency and restrictions to date remain an outrageous investigate concern.

\section{D.BEST STRATEGICAL APPROACH FOR LEARNERS WHICH COLLECTS THEIR OWN DATA}

A periphery ask about request revolves around learning structures that instead of absolutely using data accumulated by some various techniques, viably assembles 
data for its very own taking care of and learning. The assessment is surrendered to finding the best method to thoroughly give up the manage to the gaining knowledge of be counted. for example don't forget a prescription testing systemwhich endeavor to get acquainted with the achievement of the medicine while watching the revealed patients for possible cloud indications and endeavor to in this manner constraining them..

\section{E.PRIVATENESS RETAINING STATISTICS MINING}

This technique fuses enough applying facts mining and getting effects with out abusing the hid informationis pulling in direction of motion of studies frameworks and past. Consider,a medicinal examination routine trainedwith information from helpful offices any place all through the world. Notwithstanding, by virtue of security concerns, this sort of livelihoods isn't, everything considered, searched for after. In spite of whether this shows a go transversely over street between information mining and information security, propelling assessment says a framework can have both. One proposed strategy of the above issue is to build up a run of the mill learning calculation instead of a focal database. The entirety of the helpful focuses may be permitted to utilize the calculation over pre-portrayed limits to ensure the security of to the patient and consequently going with. This is an affecting examination space, joining quantifiable abuse of information and later cryptographic techniques to guarantee information security.

\section{NEVER-ENDING LEARNERS}

The greater part of the AI attempts incorporates setting up the learner using certain instructive groupings, by then putting aside the understudy and use the yield. Notwithstanding, learning in people and different creatures alter always, changing various aptitudes in development with knowledge, and utilize these learning and breaking points in an absolute synergistic way. business vocations of ML calculations, learning in computers to date had remained striking veered from learning in being or creature. An elective way of thinking that more diligentlycapture the assortment, capacity and accumulatingcharacter of learning in being, is named as interminable learning. For example, the Never Ending Language Learner (NELL) [8] is an understudy whose point of confinement is understanding how to fathom site pages and has been spoken to look at the web dependably since January 2010. NELL has obtainedalmost 80 million confirmation weighted closures (Example, servedWith(tea, scones)) and has had the decision to learn million game plans of highlights and parameters that capacitate it to get these sentiments. Also, it has gotten skilled in analyzing (disengaging) more emotions, and oust oldinaccurateones, adding to a gathering of sureness and provenance for every conviction and there by improvingeach day than the last.

\section{CATEGORISATION OF ML ALGORITHMS}

An amazing number of ML figuring have been organized and introduced over past years. Few out of every odd individual of them are comprehensively known. Some of them didn't satisfy or deal with the issue, so another was displayed in its place. Here the computations are widely amassed into two class and those two social events are further sub-isolated. This portion endeavor to name most unmistakable ML figurings and the accompanying territory takes a gander at three most for the most part used ML estimations.

\section{A. GROUP BY LEARNING STYLE}

a) Supervised getting to know - inputdata and getting ready records have a pre-selected name as an example true/fake, effective/negative, unsolicited mail/no longer unsolicited mail, and so forth. A restrict or a classifiers is made and arranged to expect the name of test information. The classifier is correctly tuned (parameter regards are adjusted)to achieve a sensible level of precision.

b) unverified getting to know - enter data or planning information is not stamped. A classifier is organized via thinking current fashions or collecting in the readiness datasets.

c) Semicoordinated culture - preparation dataset carries both checked and unlabelled records. The classifieris teach to pick up functionality with the publications to request and call the statistics similarly as to foresee.

d) Reinforcement learning - The figuring is set up to framework to condition with the objective that the prize or analysis signal is intensified. The classifier isn't modified directly to pick the movement, anyway rather arranged tofindthemost repaying exercises by experimentation.

e) Transduction - Though it has practically identical qualities with manage adjusting, yet it doesn't develop an unequivocal classifier. It attempts to anticipate the yield subject to getting ready data, planning name, and testdata.

f) Getting to learn - The classifier is trainedto learn from the inclination it incited all through beyond levels.

g) it is fundamental and successful to sift through the ML figuring regarding getting to know methods while one need to bear in mind the giganticness of the readiness facts and select the portrayal pick that supply the greater substantial stage of accuracy.

\section{B. BY SIMILARITY of ALGORITHMS group}

\section{a. Algorithms Regressions}

Backslide assessment is a bit of farsighted examination and attempts the co-association b/w subordinate (target) and free factors. The remarkable backslide models are:LinearRegression, LogisticRegression, StepwiseRegression , Ordinary Least SquaresRegression (OLSR), MultivariateAdaptive RegressionSplines (MARS) , Locally Estimated ScatterplotSmoothing (LOESS, etc.

\section{b. based Algorithms request}

Case base information version shops prevalence of having ready information in place of jogging up a specific importance of target work. At some thing point some other trouble or version is skilled, it's miles tested consistent with the set away fashions to select or are looking ahead to the purpose limit fee.it could basically override a fixed away case with the useful resource of a few different if this is an unequalled healthful than the past.

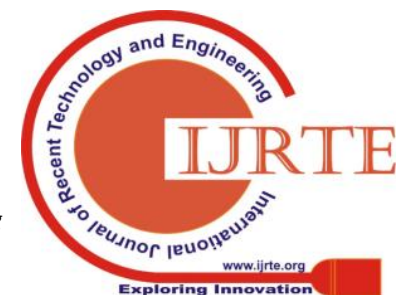




\section{Improving the Performance of the Prediction by Machine Learning Algorithms}

alongside those traces, they may be usually called champ take-all gadget. fashions: k-Nearest Neighbor (KNN), gaining knowledge of Vector Quantisation (LVQ), Self-Organizing Map (SOM), locally Weighted gaining knowledge of (LWL, and many others.

\section{c. Algorithm Regularisation}

Regularization is simplest the path towards checking overfitting or diminishing the special cases. Regularization is only a clear but lovely trade this is augmented with one of a kind present ML models customarily Regressive models. It smoothes up the backslide line by way of castigatingany wound of the curve that endeavors to prepare the irregularities. Examples:Ridge Regression, Least Absolute Shrinkage and selection Operator (LASSO), Elastic internet, Least-attitude Regression (LARS, and many others.

\section{d. selection Tree Algorithms}

A desire tree creates atree like structure together with of capacity responses for an issue challenge to particular desires. it's so namedfor it begins offevolved with a completely unique direct choice or root, which through then forks off into exceptional branches till a choice or determine is made, molding a tree. they'll be favored for its capability to formalize the hassle close to to technique that alongside the ones lines lets in perceiving capability interest plans faster and further successfully than others. fashions: type and Regression Tree (CART), Iterative Dichotomiser three (ID3), C4.five and C5.0, Chi-squared AutomaticInteraction Detection (CHAID), preference Stump, M5, Conditional desire wooden, and so on.

\section{e. Bayesian Algorithms}

A social event of ML counts use Bayes' Theorem to deal with accumulating and backslide issues.

Examples:Naive Bayes, Gaussian Naive Bayes, Multinomial Naive Bayes, Averaged One-Dependence Estimators (AODE), Bayesian notion network (BBN), Bayesian network (BN, and lots of others.

\section{f. resource Vector system (SVM)}

SVM is so renowned a ML method that it will in awesome be its very own one in each of a type social affair. Ituses a detaching hyper plane or a preference aircraft to demarcate preference limits among a ton of statistics points classified with one-of-a-kind names. it is a painstakingly coordinated path of motion depend. with the useful useful resource of the day's quit, the depend develops a extraordinary hyperplane the usage of enter statistics or getting ready records and this choice aircraft in turnscategories new fashions. thinking about the element being applied, SVM can perform each immediate and nonlinear sport plan.

\section{g. Clustering Algorithms}

Batching is forced over using permeated plan in datasets to offer an motive at the back of and stamp the facts consequently.Examples:exact enough-way, suitable sufficient-Medians, Affinity Propagation, Spectral Clustering, Ward dynamic collecting, Agglomerative packing. DBSCAN, Gaussian combinations, Birch, suggest Shift, Expectation Maximization (EM, and so forth.

\section{h. affiliation Rule analyzing Algorithms}

Alliance tips help discover correlation among reputedly unassociated statistics. they are commonly utilized by internet company agency places to anticipate client practices and future wants to elevate great drawing in topics to him. fashions: Apriori estimation, Eclat figuring, and many others.

\section{i. artificial Neural network (ANN) Algorithms}

A model reliant at the produced and errands of licensed neural frameworks of people or animals. ANNs are visible as non-direct modelsas it endeavors to discover complicated dating amongst statistics and yield data. Regardless, it draws take a look at from information in desire to thinking about the whole set and in like manner diminishing fee and time. fashions: Perceptron, again-Propagation, Hop-area community, Radial foundation function community (RBFN, and so on.

\section{j. Deep learning Algorithms}

those are regularly modernized variations of ANNs that growth by way of manner of the rich load of records in recent times.

they will be utiliseslarger neural frameworks to deal with semi-oversaw problems in which critical piece of a multiply facts is unlabelled or now not asked. fashions: Deep Boltzmann tool (DBM), Deep perception Networks (DBN), Convolutional Neural network (CNN), Stacked vehicle-Encoders, and masses of others.

\section{k. Dimensionality good deal Algorithms}

Dimensionality decline is constantly used to reduce a greater educational series to its most discriminative fragments to incorporate pertinent facts and delineate itwith less capabilities. This offers a suitable reputation for data with wonderful features or of immoderate dimensionality and helpers in completing directed portrayal more correctly.Examples: number one thing assessment (PCA), primary detail Regression (PCR), Partial Least Squares Regression (PLSR), Sammon Mapping, Multidimensional Scaling (MDS), Projection Pursuit, Linear Discriminant evaluation (LDA), combination Discriminant evaluation (MDA), Quadratic Discriminant evaluation (QDA), flexible Discriminant evaluation (FDA, and lots of others.

\section{Ensemble Algorithms}

The crucial concept the use of a social affair method is to arrange the projections of multiple flimsier estimator which can be independently organized which will help up or enhance generalisability or quality over a lone estimator. The types of understudies and the outstanding technique to combine them is carefully picked as to heighten the accuracy. models: Boosting, Bootstrapped Aggregation (Bagging), AdaBoost, Stacked Generalization (blending), Gradient Boosting Machines (GBM), Gradient Boosted Regression bushes (GBRT), Random forest, extraordinarily Randomized timber, and masses of others. 


\section{Measuring and Comparing Performance Of Famous Ml Algorithms}

Regardless of the manner by which that different specialists have added to ML and various estimations and systems have been presented as referenced starting at now, on the off chance that it is unflinchingly analyzed a gigantic piece of the certified ML method joins 3 sizeable oversaw figuring or their series. those three are to be unique, Naive Bayes, help Vector device and selection Tree. A first rate deal of bosses have used the credibility of those three, be it sincerely or with a boosting test to beautify the restrict further. those three joins are talked about fast within the going with place.

\section{A.NAIVE BAYES CLASSIFIER}

it's far a directed request method developed using Bayes' Theoremof prohibitive possibility with an 'Unsuspecting' assumption that every pair of function is generally self-governing. that is, in grade by grade direct phrases, proximity of a problem isn't inspired with the beneficial resource of closeness of a few exceptional using any techniques. regardless of this over-streamlined supposition, NB classifiers finished thoroughly in numerous useful conditions, as in content cloth accumulating and junk mail vicinity. handiest a restricted quantity of getting prepared statistics is needto take a look at certain parameters. near, NB classifiers have amazingly beated even appreciably pushed sport plan techniques.

\section{B. GUIDE VECTOR MACHINE}

SVM, a few other managed portrayal estimation proposed with the aid of manner of Vin Sixties have beginning late pulled in a noteworthy concept of researchers. The smooth geometrical explanation of this method consists of determining an fine confining plane or hyperplane that separates the 2 commands or lots of facts concentrates impartially and is equidistant from them . SVM was define that first for right now dissemination of facts facilities. A quick time later, the bit restrict changed into familiar with address non-right away snippets of statistics as properly.

\section{C.CHOICE TREE}

Over-gathering countering over counter tree, appreciably referred to as preference tree is a standout among one in every of a kind controlled reading rely amount. It assembles a chart or tree that uses fanning framework records every probable result of a desire. In a diffusion tree depiction, each internal middle aspect tests a phase, each branch identifies with very last consequences of over-the-counter counter determine center and each leaf quit doles out over-over-the-counter-counter over over-the-counter counter splendor mark. To installation a case, a top-down method is completed begin at the counter of tree. For a specific segment or center, branch concurring to estimation of over the counter counter information element for that tremendous is taken into consideration till a leaf is come to or a call is picked.

via use of and thru, over the counter counter presentations of overover counter three had been for over-overover over the counter counter maximum detail differentiated overover counter a notable deal of tweets and tests terrific, terrible and honest. The unrefined tweets were taken from Sentiment140 instructive collection. with beneficial aid of over-over the counter-countern thoseover counter are pre-prepared and stamped overover over-the-counter counter a python software software program software. counter classifier have been displayed to same data. equal figuring of function desire, dimensionality cut price and ok-overlay endorsement had been used for every situation. The estimations were taken a gander at reliant at the planning time, desire time and exactness of over the counter gauge. The take a look at final results is given under.

\section{PACKAGES}

One easy signal of movement in ML is its critical certifiable applications, a number of which might be right now delineated here.it's far to be visible that until 1985 there was no significant corporation makes use of of ML computations.

\section{A. SPEECH recognition}

All gift talk affirmation structures to be had over-over the counter-counterover overover over the counter counter marketplace use AI strategies to control teach over-overover over-the-counter counter machine for better exactness. essentially, a large phase of such structures comprehend gaining knowledge of in particular levels: pre-delivery speaker-self-ruling getting equipped and put up-delivery speaker-subordinate planning.

\section{B. laptop imaginative and prescient.}

big piece over-over-the-counterover overover the counter-counterse days imaginative and prescient systems, e.g., facial confirmation programming ventures, systems prepared for custom designed workout plan minor pics of cells, use AI strategies for better exactness. for example, overover over-the-counter counter publish place of business makes use of a laptop vision structure with a handwriting analyser thusly organized to kind letters with bodily composed areas usually with a precision diploma as immoderate as $85 \%$.

\section{BIO-SURVEILLANCE}

Severalgovernment physical sports to pursue doable flare-americaof diseasesuses ML counts. recall over-overover over the counter counter RODS adventure in western Pennsylvania. This task accumulates attestations reviews to emergency rooms over-medicinal capsules' buy information to offer extra training data. Multifaceted nature of this type of unexpected and dynamic enlightening assortments may be managed gainfully usage of over counter motorized studying strategies so to speak.

\section{D. robotic OR AUTOMATION control}

ML strategies are, because it were, applied in robot and automated systems. as an instance, endure in thoughts overover over-the-counter counter counter ML to benefit manage techniques for strong flight and aerobatics of helicopter. 


\section{Improving the Performance of the Prediction by Machine Learning Algorithms}

Oneself driving vehicles made via Google usesML to devise from scene records.

\section{E. EMPIRICAL technology EXPERIMENTS}

A brilliant social event information focused technological bdd5b54adb3c84011c7516ef3ab47e54 disciplines use ML techniques in a couple of it asks about. for example, ML is being found out in innate developments, to understand ordinary amazing topics in location science, and in Neuroscience and mental exam.

overover over the counter counter little scale but noteworthy utilization of ML includes unsolicited mail keeping apart, blackmail revelation, task identity and insightful exam (e.g., atmosphere bet, protections trade preference, publicize take a look at, and so on.).

\section{FUTURE SCOPE}

Computer based intelligence is investigate locale that has pulled in a huge amount of awe inspiring characters and it can reveal further. information, the three maximum vital destiny sub-problems are picked to be mentioned proper right right here.

\section{A.EXPLAINING BEING gaining knowledge of}

A referenced previously, AI theories have been perceivedfitting to comprehendfeatures of gaining knowledge of in people and animals. Bolster analyzing computations test the dopaminergic neurones impelled sports activities in animals within the course of reimbursement primarily based completely reading with super accuracy. ML computations for uncovering sporadicdelineations of often displaying up snap shots are looking forward to visible competencies perceived in animals' critical seen cortex. Regardless, the massive drivers in being or animal analyzing like instigation, loathsomeness, criticalness, starvation, regular sports activities and studying with the resource of experimentation over awesome time scales, are not but considered in ML estimations. This a potential opportunity to find out an undeniably summarized concept of locating that entailsboth animals and gadget.

\section{B.PROGRAMMING LANGUAGES CONTAINING device studying PRIMITIVES}

In majority of makes use of, ML computations are intertwinedwith bodily coded programs as a part of an software software programming. The want of some different programming language this is self maintaining to assist bodily created subroutines in addition as those defined as "to gain information of." it could allow the coder to define a set of information belongings yields of each "to be insightful" software program and pick a figuring from the social occasion of primary reading techniques already gave within the language. Programming lingos like Python (Sckit-research), $\mathrm{R}$, and so forth already the use of this notion in greater diminutive increase. Regardless, a charming new query is raised as to developa version to outline applicable analyzing experience for every subroutine marked as "to be gotten the draw close of", timing, and securityin case ofAny unforeseenmodification to this gadget'sfunction.

\section{C.perception}

A consolidated idea of pc perception that can relate ML consists of a are used mainly shape of computer astuteness these days together with facts now not limited to especially driven imaginative and prescient, communicate request, and so on., is each different functionality research vicinity. One belief provoking problem is the coordination of different senses (e.g., discover, listen, touch, and masses of others) to set up a shape which use independently directed seeing a manner to observe one smooth knowledge using the others. Researches in developmental cerebrum discover have stated reliably affordable reading in beings when various input modalities are given, and thinks approximately on co-getting equipped structures insinuate similar results.

\section{Conclusion}

The foremost target of ML investigators is to format logically capable (to the volume whenever and location)and practical all spherical precious gaining knowledge of strategies that may carry out improved over an expansive country. concerning ML, the viability by a methodology uses records belongings this is in like way a large creation angle close by presence multifaceted layout. better precision of preference and beingly interpretable determine rules are in like way of excessive criticalness.

Being without a doubt data driven or has the choice to get a gander at a high-quality deal of records in greater diminutive among times of time, ML counts has an aspect over guide or direct programming. furthermore they may be tons of the time logically precise and no longer slanted to being tendency. keep in mind the going with circumstances:

Headway of an object to enlighten acumen endeavors the use of sensors, like communicate affirmation, laptop imaginative and prescient, and many others. It is simple for simply every body to name an image of a letter via manner of the letters all collectively it approach, except arranging a computation to play out this mission is inconvenient.

Customisation of an object as proven with the resource of the usage of the earth it's far surpassed directly to. recollect, speak affirmation program ventures that must be changed through the requirements of the patron. Like internet organization goals that modifications the topics regarded through the usage of customers or $d$ peruser that engages e-mail location as indicated through way of patron inclinations. Direct programming would not have the potential to change when introduced to distinct scenario.

ML providesa programming the electricity and flexibility at the same time as superb. notwithstanding some software (e.g., to create set up duplication applications) in which ML also can dismiss to be useful, with addition of records assets and developing call for in modified custom isable programming, ML will thrive in no longer all that a protracted way off destiny. apart from programming improvement, ML will probably information assist reform the general outlook of pc generation. by means of the use of converting the portraying question from "a manner to software program a computer" to "the way to empower it to software application itself," ML orders the headway of devices that the self-looking, self-diagnosing and self-fixing, and the employments of the information circulate available inside a program in preference to essentially setting it up.

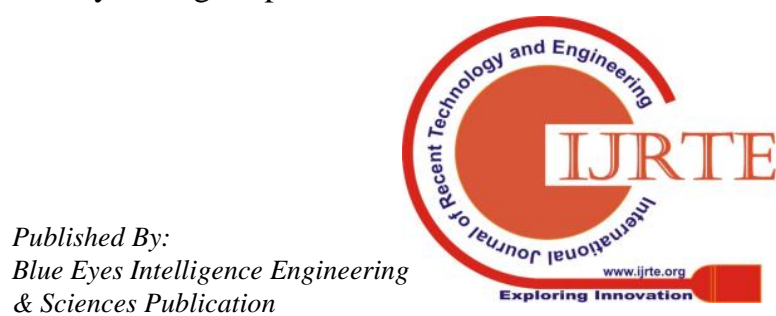


So additionally, it will assist change Statistical requirements, by way of providing more computational role. obviously, the two information and computer technological bdd5b54adb3c84011c7516ef3ab47e54 will in like manner decorate ML as they make and contribute more advanced theories to change the technique for studying.

\section{REFERENCES}

1. T. M. Mitchell, Machine Learning, McGraw-Hill International, 1997.

2. T.M. Mitchel, The Discipline of Machine Learning, CMU-ML-06-108, 2006

3. N. Cristianini and J. Shawe-Taylor. An Introduction to Support Vector Machines. Cambridge University Press, 2000.

4. E. Osuna, R. Freund, and F. Girosi. Support vector machines: training and applications. AI Memo 1602, MIT, May 1997.

5. V. Vapnik. Statistical Learning Theory. John Wiley \& Sons, 1998

6. C.J.C. Burges. A tutorial on support vector machines for pattern recognition. Data Mining and Knowledge Discovery, 2(2):1-47, 1998.

7. Taiwo Oladipupo Ayodele, Types of Machine Learning Algorithms, New Advances in Machine Learning, Yagang Zhang (Ed.), InTech, 2010

8. T. Mitchell, W. Cohen, E. Hruschka, P. Talukdar, J. Betteridge, A. Carlson, B. Dalvi, M. Gardner, B. Kisiel, J. Krishnamurthy, N. Lao, K. Mazaitis, T. Mohamed, N. Nakashole, E. Platanios,A. Ritter, M. Samadi, B. Settles, R. Wang, D. Wijaya, A. Gupta, X. Chen, A. Saparov,M. Greaves, J. Welling, Never-Ending Learning, Proceedings of the Twenty-Ninth AAAI Conference on Artificial Intelligence, 2014

9. Pedregosa et al.,Scikit-learn: Machine Learning in Python, JMLR 12 pp. 2825-2830, 2011

10. Wang, J. and Jebara, T. and Chang, S.-F. Semi-supervised learning using greedy max- cut.Journal of Machine Learning Research Volume 14(1), 771-800 2013

11. Chapelle, O. and Sindhwani, V. and Keerthi, S. S. Optimization Techniques for Semi- Supervised Support Vector Machines, Journal of Machine Learning Research , Volume 9, 203-233, 2013

12. J. Baxter. A model of inductive bias learning. Journal of Artificial Intelligence Research, 12:149-198, 2000.

13. S. Ben-David and R. Schuller. Exploiting task relatedness for multiple task learning. In Conference on Learning Theory, 2003.

14. W. Dai, G. Xue, Q. Yang, and Y. Yu, Transferring Naive Bayes classifiers for text classification.AAAI Conference on Artificial Intelligence, 2007.

15. H. Hlynsson. Transfer learning using the minimum description length principle with a decision tree application. Master's thesis, University of Amsterdam, 2007.

16. Z. Marx, M. Rosenstein, L. Kaelbling, and T. Dietterich. Transfer learning with an ensemble of background tasks. In NIPS Workshop on Transfer Learning, 2005.

17. R Conway and D Strip, Selective partial access to a database, In Proceedings of ACM Annual Conference, 85 - 89, 1976

18. P D Stachour and B M Thuraisingham Design of LDV A multilevel secure relational database management system, IEEE Trans. Knowledge and Data Eng., Volume 2, Issue 2, 190 - 209, 1990

19. R Oppliger, Internet security: Firewalls and beyond, Comm. ACM, Volume 40, Issue 5, 92 -102, 1997

20. Rakesh Agrawal, Ramakrishnan Srikant, Privacy Preserving Data Mining, SIGMOD '00 Proceedings of the 2000 ACM SIGMOD international conference on Management of data, Volume 29 Issue 2,Pages 439-450, 2000

21. A. Carlson, J. Betteridge, B.Kisiel, B.Settles,E. R.Hruschka Jr,and T. M. Mitchell, Toward an architecture for never-ending language learning, AAAI, volume 5, 3, 2010

22. X. Chen, A. Shrivastava, and A. Gupta, Neil: Extracting visual knowledge from web data, In Proceedings of ICCV, 2013.

23. P. Donmezand J. G. Carbonell, Proactive learning: cost-sensitive active learning with multiple imperfect oracles. In Proceedings of the 17th ACM conference on In- formation and knowledge management, 619-628. ACM, 2008

24. T. M.Mitchell, J. Allen, P. Chalasani, J. Cheng, O. Etzioni, M. N. Ringuetteand J. C. Schlimmer, Theo: A framework for self-improving systems, Arch. for Intelli- gence 323-356, 1991

25. Gregory, P. A. and Gail, A. C. Self-supervised ARTMAP Neural Networks, Volume 23, 265-282, 2010

26. Cour, T. and Sapp, B. and Taskar, B. Learning from partial labels, Journal of Machine Learning Research, Volume 12, 1501-1536 2012
27. Adankon, M. and Cheriet, M. Genetic algorithm-based training for semi-supervised SVM, Neural Computing and Applications, Volume 19(8), 1197-1206, 2010 